\title{
Universality of the one dimensional Bose gas with delta interaction
}

\author{
Luigi $\operatorname{Amico}^{(1)}$ and Vladimir Korepin ${ }^{(2)}$ \\ (1) MATIS-INFM \& Dipartimento di Metodologie Fisiche e Chimiche (DMFCI), \\ Universitá di Catania, viale A. Doria 6, I-95125 Catania, Italy and \\ (2) C.N. Yang Institute for Theoretical Physics, State University of New York at Stony Brook, NY 11794-3840
}

\begin{abstract}
We consider several models of interacting bosons in a one dimensional lattice. Some of them are not integrable like the Bose-Hubbard others are integrable. At low density all of these models can be described by the Bose gas with delta interaction. The lattice corrections corresponding to the different models are contrasted.
\end{abstract}

\section{INTRODUCTION}

The Bose gas with delta interaction is one of the most famous models for strongly interacting bosons [1, 2] (the corresponding classical dynamics is described by the non-linear Schrödinger equation). In one dimension this model possesses infinitely many, mutually commuting integrals of the motion and is therefore called integrable. The integrability of the Bose gas can be formulated within the Quantum Inverse Scattering Method (QISM) 3] that exploit a crucial property of the twobody scattering matrix of the system: it satisfy the YangBaxter equation. This reduces the many-body dynamics to a two body dynamics. In the Appendix we explain the QISM \& the algebraic Bethe Ansatz [it uses a transfer matrix]. In the last decades many authors studied integrable lattice regularization of the continuous Bose gas [4, 5, 6, 7, 8, 9, 10, 11, 12, 13, 14]. The procedure it is not straightforward since a direct quantization of lattice classical integrable theory whose continuous limit is the classical continuous Bose gas field theory leads to "nonlocal" Hamiltonians (that is the interaction involves far neighbors). This inconvenience was circumvented in two, somewhat complementary ways: keep the scattering matrix of the continuous theory unaltered and change the transfer matrix and then the Hamiltonian 4, 5, 6, 7]; alternatively, change the scattering matrix keeping the Hamiltonian formally unaltered [8, 9]. In both cases, however, the Hamiltonians though (quasi) local, involve higher order processes whose physics is hard to read-out.

Another paradigmatic lattice model for interacting bosons is the Bose-Hubbard model. It was proposed first by Haldane [15] (see also 16]), and widely used in many different contexts. In mesososcopic physics it describes granular superconductors, Helium films, and Josephson junctions arrays 17. In quantum optics, the Bose-Hubbard dynamics was proposed to model bosonic atoms in optical lattices [18] and as effective theory for higher spin chains [19]. The Bose-Hubbard Hamiltonian describes charged bosons hopping in a $d$-dimensional lattice and experiencing a Coulomb repulsion. The resulting competition between kinetic and electrostatic energies in- duces quantum fluctuations that dominate the zero temperature phase diagram. At commensurate number of bosons per site the Bose-Hubbard model predicts a Mott insulator-superfluid quantum phase transition controlled by the ratio between the bosonic tunneling rate $t$ and the Coulomb repulsion. In optical applications the system parameters are tuned by changing the laser intensity 20]; the criticality of the superfluid-insulator quantum phase transition is substantially altered by the confining harmonic potential 21].

In this paper we focus on one dimension. We remark that $d=1$ is more than an academic case for the physical systems captured by the models under consideration. In fact bosonic chains are commonly realized both with optical confinement [20] and, using a different technology, with Josephson junctions [17. Quantum effects are strongest in the one-dimensional case. For example, in the dilute limit of optical lattices quantum many body effects cause the breakdown of the Gross-Pitaevskii theory (valid for $3 d$ systems) and drive the system to the so called "Tonks-Girardeau" regime 22$]$ where the exact results of Lieb and Liniger are crucial 23]. Exact results of the Bose-Hubbard model would be desirable to study physical systems like the above ones, at higher densities. However the exact solution of the one dimensional Bose-Hubbard model is still a challenging problem. For infinite range hopping the thermodynamics is fully established 25]. For nearest neighbor hopping, the coordinate Bethe ansatz fails 24] due to the multi-occupancy characteristic of the bosonic statistics [26]. Recent numerical studies suggested that the distribution of level spacings obeys Wigner-Dyson statistics 27], this considered as indication of non-integrability [28].

In this paper we discuss the integrable continuous field theory of the Bose gas as universal low density limit of various integrable (Faddeev-Takhtadjan-Tarasov 7], Izergin-Korepin [4], and quantum Ablowitz-Ladik 8, $9,12,13,14]$ ) and non integrable (Bose-Hubbard) lattice models. In particular a number of results obtained for the Bose gas can be used for the behavior of the systems phrased by Bose-Hubbard models. Finally we discuss the differences between the existing integrable lat- 
tice regularization of the Bose gas and the non-integrable Bose-Hubbard model.

The paper is laid out as follow. In the next section we summarize a number of properties of the integrable field theory of the Bose gas. In section III we apply some of these properties to the Bose-Hubbard model. The lattice integrable theories are discussed in section IV. In section $\mathrm{V}$ we draw our conclusions. In the Appendix we summarize the main ideas of the QISM.

\section{THE BOSE GAS WITH DELTA INTERACTION}

We shall start with continuous field theory. This will be the continuous limit of all bosonic models considered in this paper. The one dimensional Bose gas is described by an integrable non-relativistic model of field theory 1 , 3. The Hamiltonian is:

$$
\mathcal{H}_{B G}=\int d x\left[\left(\partial_{x} \Psi^{\dagger}\right)\left(\partial_{x} \Psi\right)+c \Psi^{\dagger} \Psi^{\dagger} \Psi \Psi-h \Psi^{\dagger} \Psi\right] .
$$

Here: $\left[\Psi(x), \Psi^{\dagger}(y)\right]=\delta(x-y)$ and $\left[\Psi^{\dagger}(x), \Psi^{\dagger}(y)\right]=0$. The coupling constant is denoted by $c$, and $h$ is the chemical potential. It is equivalent to a many body quantum mechanical problem with Hamiltonian:

$$
H_{B G}=-\sum_{j=1}^{N} \frac{\partial^{2}}{\partial z_{j}^{2}}+2 c \sum_{N \geq j>k \geq 1} \delta\left(z_{j}-z_{k}\right) .
$$

The energy is $E=\sum_{j=1}^{N}\left(u_{j}^{2}-h\right)$; the momenta $u$ 's obey Bethe equations:

$$
e^{i u_{j} L}=\prod_{k \neq j}^{N} \frac{u_{j}-u_{k}+i c}{u_{j}-u_{k}-i c} \quad j=1 \ldots N
$$

Let us consider repulsive case $c>0$. In the thermodynamic limit $N, L \rightarrow \infty$ such that $N / L=D=$ const the ground state energy-density [per unit length] is

$$
E=\int_{-q}^{q} \epsilon_{0}(u) \rho(u) d u=\int_{-q}^{q}\left(u^{2}-h\right) \rho(u) d u
$$

The function $\rho(u)$ describes the distribution of momenta in the ground state. It is defined by the equation:

$$
2 \pi \rho(u)=1+\int_{-q}^{q} K(u-v) \rho(v) d v \doteq \mathcal{Z}(u),
$$

where $\mathcal{Z}(u)$ is called dressed charge. The kernel of the integral equation is $K(x)=2 c /\left(c^{2}+x^{2}\right)$. For further purposes it is convenient to define a function $\tau(u)$ through

$$
2 \pi \tau(u)=K(u-q)-\int_{-q}^{q} d v K(u-v) \tau(v) .
$$

The density, given by $D=\int_{-q}^{q} \rho(u) d u$, depends on the chemical potential since $q=q(h)[3]$. The Bose gas is characterized by different phases depending on the sign of the coupling constant $c$. Let us describe the ground state at zero temperature:

i) For $c=0$ we have free bosons. In the ground state all the particles have zero momentum (Bose-condensation).

ii) In the repulsive case $c>0$ the Pauli principle is valid (this is the distinctive feature of the one dimensional bosons). The ground state is a Fermi sphere.

iii) In the attractive case $c<0$ the ground state is a single large bound state of all the particles. All the particles stay close to one another (in configuration space). It is a droplet. One can say that in this case the phases separate.

In the repulsive case at zero temperature the asymptotics (large distances) of correlation functions is [3, 29]

$$
\begin{aligned}
& \left\langle\Psi^{\dagger}(x) \Psi(0)\right\rangle=A x^{-1 / \theta} \\
& \left\langle\Psi^{\dagger}(x) \Psi(x) \Psi^{\dagger}(0) \Psi(0)\right\rangle=D^{2}+\frac{A}{x^{2}}+B \frac{\cos [2 \pi D x]}{x^{\theta}}
\end{aligned}
$$

where the critical exponent $\theta$ depends on the density in a remarkably simple way through:

$$
\theta=2 \mathcal{Z}^{2}(q)=4 \pi D / v_{F}
$$

The thermal correlation functions can be obtained by replacing: $x \rightarrow \frac{v_{F}}{\pi T} \sinh \left(\pi T x / v_{F}\right)$ in these expression. Here $v_{F}$ is the Fermi velocity [for the model under consideration, it coincides with the velocity of sound]. So in the repulsive case the asymptotic of the thermal correlation functions is

$$
\begin{aligned}
& \left\langle\Psi^{\dagger}(x) \Psi(0)\right\rangle_{T}=\left(\frac{v_{F}}{\pi T}\right)^{-1 / \theta} \exp \left(-\pi T x / v_{F} \theta\right) \\
& \left\langle\Psi^{\dagger}(x) \Psi(x) \Psi^{\dagger}(0) \Psi(0)\right\rangle_{T}=B_{2}\left(\frac{2 \pi T}{v_{F}}\right)^{2} e^{-2 \pi T x / v_{F}} \\
& +B_{3}\left(\frac{2 \pi T}{v_{F}}\right)^{\theta} e^{-\pi T \theta x / v_{F}} \cos (2 \pi D x)
\end{aligned}
$$

The coefficients $A, B, B_{2}, B_{3}$ are related to certain form factors [29].

\section{APPLICATION TO THE BOSE-HUBBARD MODELS}

The Hamiltonian for the one dimensional BoseHubbard model reads

$$
H_{B H}=\sum_{i=-N_{s}}^{N_{s}}\left[U\left(n_{i}-1\right)-\mu\right] n_{i}-t\left(a_{i}^{\dagger} a_{i+1}+a_{i+1}^{\dagger} a_{i}\right)
$$

where the operators $n_{i}:=a_{i}^{\dagger} a_{i}$ count the number of bosons at the site $i$; operators $a_{i}, a_{i}^{\dagger}$ obey the canonical commutation relations $\left[a_{i}, a_{j}^{\dagger}\right]=\delta_{i j}$ and and $2 N_{s}+1$ 
is the number of sites. The parameters $t, U$ of (8) are the hopping amplitude and the strength of the on-site Coulomb repulsion, respectively, while the chemical potential $\mu$ fixes the average number of bosons in each site. The density of bosons in the lattice is given by $D=N /\left(2 N_{s}+1\right) \Delta$. Here $\Delta$ is the lattice spacing. The Bose-Hubbard model can be considered as a possible lattice regularization of the Bose gas. In fact at small filling factor $\nu=N /\left(2 N_{s}+1\right)=D \Delta$ the Bose-Hubbard model can be described by the Bose gas with delta interaction. In order to obtain the correct commutators, in the continuous limit $\Delta \rightarrow 0$ one has to re-normalize the operators: $a_{i}=\sqrt{\Delta} \Psi(x)$ and $n_{i}=\Delta \Psi^{\dagger}(x) \Psi(x), x=\Delta i$. The Bose-Hubbard model then reduces to the Bose gas

$$
H_{B H}=t \Delta^{2} H_{B G},
$$

with $c=U /(t \Delta)$ and $h=-(\mu+2 t) /\left(t \Delta^{2}\right)$. Therefore results for the Bose gas can be phrased for systems captured by the Bose-Hubbard model. The asymptotics of the zero-temperature correlations in the repulsive case are

$$
\begin{aligned}
\left\langle a_{i}^{\dagger} a_{j}\right\rangle & =A(i-j)^{-1 / \theta}, \\
\left\langle n_{i} n_{j}\right\rangle & =\left(\frac{\nu}{\Delta}\right)^{2}+\frac{A}{(i-j)^{2}}+B \frac{\cos [2 \pi \nu(i-j) / \Delta]}{(i-j)^{\theta}}
\end{aligned}
$$

where the critical exponent $\theta$ is

$$
\theta=2\left(1+\frac{4 D}{c}\right)=2\left(1+\frac{4 \nu t}{U}\right) .
$$

The thermal correlation functions read

$$
\begin{aligned}
\left\langle a_{i}^{\dagger} a_{j}\right\rangle_{T} & =\left(\frac{v_{F}}{\pi T}\right)^{-1 / \theta} \sinh \left[\pi T(i-j) / v_{F}\right]^{-1 / \theta} \\
\left\langle n_{i} n_{j}\right\rangle_{T} & =B_{2}\left(\frac{2 \pi T}{v_{F}}\right)^{2} e^{-2 \pi T(i-j) / v_{F}} \\
& +B_{3}\left(\frac{2 \pi T}{v_{F}}\right)^{\theta} e^{-\pi T \theta(i-j) / v_{F}} \cos [2 \pi \nu(i-j) / \Delta] .
\end{aligned}
$$

\section{INTEGRABLE LATTICE MODELS}

In this section we discuss the integrable bosonic theories arising as quantum lattice regularization of the field theory of the Bose gas with delta interaction. From a classical side the Hamiltonian structure [action-angle variables] is directly related to the $R$-matrix. This constituted motivation to define the lattice models keeping unaltered the $R$-matrix (for an alternative way to construct lattice regularization of the Bose gas see the Appendix [8, 9]). Pursuing a quantum version of this procedure, namely directly quantizing the classical Lax matrices, it turns out that the arising Hamiltonians are nonlocal. Here we should note that non-local Hamiltonians are interesting by them-self: good examples are CalogeroSutherland, Haldane-Shastry [30], and pairing 31, 32, 33. models. To obtain local Hamiltonians the Lax matrices have to be modified. This modification induces complications on the final form of the though local Hamiltonians (see the Refs. 4, 5, 6, 7]). All these are solvable by algebraic Bethe Ansatz (see Ref. [3]); some basic formulas of the QISM are summarized in Appendix. Here we will present these Hamiltonians. Some of them simplify in the weak coupling limit. In this limit the resulting Hamiltonians are compared with the Bose-Hubbard model.

\section{Izergin-Korepin models}

The first integrable version of the Bose gas on the lattice was constructed in Refs [4, 5]. To write the Hamiltonian of the model in a compact way we introduce the following notations. The bosons interact differently in odd and even lattice sites. Let us start with canonical Bose operators

$$
\left[a_{j}, a_{k}^{\dagger}\right]=\delta_{k}^{j} \quad ; \quad a_{j}|0\rangle=0 .
$$

In order to describe the model, it is convenient to introduce 'renormalized' operators

$$
b_{j}=a_{j} \sigma_{j}^{-1} .
$$

Here $\sigma_{j}$ is slightly different in odd and even lattice sites:

$$
\sigma_{j}= \begin{cases}\sqrt{1+\omega a_{j}^{\dagger} a_{j}} & \text { if } j \text { is even }, \\ \sqrt{1+\omega\left(a_{j}^{\dagger} a_{j}-1\right)} & \text { if } j \text { is odd } .\end{cases}
$$

where $\omega=c \Delta / 4$. We can express everything is terms of 'renormalized' operators $b_{j}$ :

$$
\begin{aligned}
a_{j}^{\dagger} a_{j} & =b_{j}^{\dagger} b_{j}\left\{1-\omega b_{j}^{\dagger} b_{j}\right\}^{-1}, \\
\sigma_{j}^{2} & =\left\{1-\omega b_{j}^{\dagger} b_{j}\right\}^{-1} \text { for } j \text { even },
\end{aligned}
$$

For odd $j$ the expressions are slightly different:

$$
\begin{aligned}
a_{j}^{\dagger} a_{j} & =(1-\omega) b_{j}^{\dagger} b_{j}\left\{1-\omega b_{j}^{\dagger} b_{j}\right\}^{-1}, \\
\sigma_{j}^{2} & =(1-\omega)\left\{1-\omega b_{j}^{\dagger} b_{j}\right\}^{-1} \text { for } j \text { odd } .
\end{aligned}
$$

We can also write a closed commutation relations for renormalized operators:

$$
\begin{aligned}
b_{j}\{1 & \left.-\omega b_{j}^{\dagger} b_{j}\right\}^{-1} b_{j}^{\dagger}-\left\{1-\omega b_{j}^{\dagger} b_{j}\right\}^{-\frac{1}{2}} b_{j}^{\dagger} b_{j}\left\{1-\omega b_{j}^{\dagger} b_{j}\right\}^{-\frac{1}{2}} \\
& = \begin{cases}1 & \text { if } j \text { is even } \\
(1-\omega)^{-1} & \text { if } j \text { is odd } .\end{cases}
\end{aligned}
$$

In different lattice sites operators $b_{j}$ commute. Now we can present the Hamiltonian of the model as

$$
H_{I K}=-\frac{4}{3 c \Delta^{3}} \sum_{j}\left(t_{j}+t_{j}^{\dagger}+\frac{2-\omega}{2(1-\omega)}-\frac{\Delta a_{j}^{\dagger} a_{j}}{1-\omega^{2}}\right) .
$$


Below, we shall represent operators $t_{j}$ in the form

$$
t_{j}=-\frac{1}{2} O_{j}^{-1} C_{j} O_{j}
$$

The formula for operators $O$ and $C$ are different for odd and even lattice sites. For $j$ odd:

$$
\begin{aligned}
O_{j}= & \sigma_{j+1}\left\{1+\omega b_{j+1}^{\dagger} b_{j+2}\right\} \sigma_{j+2}, \\
C_{j}= & \sigma_{j}^{-1}\left\{1+\omega b_{j-1}^{\dagger} b_{j}\right\}^{-1} \sigma_{j-1}^{-2}\left\{1+\omega b_{j}^{\dagger} b_{j-1}\right\}^{-1} \times \\
& \sigma_{j}^{-1} \sigma_{j-1}\left\{1-\omega b_{j-1}^{\dagger} b_{j+1}\right\} \sigma_{j+1}, .
\end{aligned}
$$

For $j$ even:

$$
\begin{aligned}
O_{j}= & \sigma_{j-2}\left\{1+\omega b_{j-2}^{\dagger} b_{j-1}\right\} \sigma_{j-1} \\
C_{j}= & \sigma_{j-1}^{-1}\left\{1+\omega b_{j}^{\dagger} b_{j-1}\right\}^{-1} \sigma_{j}^{-2}\left\{1+\omega b_{j-1}^{\dagger} b_{j}\right\}^{-1} \times \\
& \left\{1-\omega b_{j-1}^{\dagger} b_{j+1}\right\} \sigma_{j+1} .
\end{aligned}
$$

In the weak coupling limit the Hamiltonian describes a coupling of five neighbors lattice sites $j-2 \ldots j+2$. Retaining contributions up to second order in $c \Delta$ it has the form

$$
\begin{gathered}
H_{I K}=-\frac{4}{3 c \Delta^{3}} \sum_{j} \frac{c \Delta}{8}\left(K_{j, j-1}-n_{j}-K_{j-1, j+1}\right) \\
+\frac{(c \Delta)^{2}}{16}\left(K_{j, j-1}^{2}+h_{j}\right), \\
h_{j}=\sum_{\alpha, \beta=-2}^{2}\left[v_{\alpha \beta} n_{j+\alpha} n_{j+\beta}+w_{\alpha \beta} a_{j+\alpha} a_{j+\beta}^{\dagger}\right. \\
\left.+\left(t_{\alpha}+q_{\alpha} n_{j+\alpha}\right)\left(r_{\beta} a_{j+\beta} a_{j+\beta-1}^{\dagger}+s_{\beta} a_{j-\beta} a_{j+\beta}^{\dagger}\right)\right]+ \text { h.c. }
\end{gathered}
$$

where $K_{l, m}=a_{l} a_{m}^{\dagger}+a_{m} a_{l}^{\dagger}$ (with certain coefficients $\left.v_{\alpha \beta}, w_{\alpha \beta}, t_{\alpha \beta}, q_{\alpha \beta}\right)$. After simple manipulations Eq. (28) can be written as

$H_{I K}=H_{B H}-g \frac{c \Delta}{8} K_{j-1, j+1}+g\left(\frac{c \Delta}{4}\right)^{2}\left[K_{j, j-1}^{2}+h_{j}\right]$,

where periodic boundary conditions have been considered:

$$
\sum_{j} n_{j}=\sum_{j} n_{j+1} \quad \text { and } \quad \sum_{j} n_{j}^{2}=\sum_{j} n_{j+1}^{2}
$$

the parameters in $H_{B H}$ above are $\mu / g=(c \Delta)(1-$ $5 c \Delta / 16) / 8, \quad U / g=5(c \Delta)^{2} / 128, t / g=c \Delta / 8$ with $g=-4 /\left(3 c \Delta^{3}\right)$. The Bose-Hubbard model differs from Itzergin-Korepin model in the presence of the quadratic hopping and in the non local terms. In the continuous limit, when the lattice spacing $\Delta \rightarrow 0$, this lattice model turns into a continuous Bose gas (1).

\section{Faddeev-Takhtadjan-Tarasov models}

In this section we discuss another lattice integrable version of the Bose gas. The Hamiltonian was suggested by Faddeev-Takhtadjan-Tarasov in Ref. [7]. The model has the same $R$-matrix as the continuous Bose gas. It looks like a quantum spin chain with negative spin. Let us first introduce an operator of 'angular-momentum' $J_{j, j+1}$. It is defined as a solution of the following operator equation:

$$
J_{j, j+1}\left(J_{j, j+1}+1\right)=2 S_{j} \otimes S_{j+1}+2 s(s+1) .
$$

Here the 'spin' is $s=-2 /(c \Delta)$. The Hamiltonian of the Faddeev-Takhtadjan-Tarasov model is

$$
H_{F T T}=-2 \kappa \sum_{j} \Gamma^{\prime}\left(J_{j, j+1}+1\right) / \Gamma\left(J_{j, j+1}+1\right) .
$$

The relation with the lattice bosons becomes transparent using the Holstein-Primakov realization of spins:

$$
\begin{aligned}
S_{j}^{x} & =\frac{a_{j}^{\dagger} \rho_{j}+\rho_{j} a_{j}}{\sqrt{c \Delta}}, \\
S_{j}^{y} & =i \frac{-a_{j}^{\dagger} \rho_{j}+\rho_{j} a_{j}}{\sqrt{c \Delta}}, \\
S_{j}^{z} & =-\frac{2}{c \Delta}\left(1+\frac{c \Delta a_{j}^{\dagger} a_{j}}{2}\right),
\end{aligned}
$$

with $\rho=\sqrt{1+c \Delta a^{\dagger} a / 4}$. For small $c$, the spin $s$ is large. The Hamiltonian simplifies (second order in $1 / s$ ):

$$
\begin{gathered}
H_{F T T}=\frac{\kappa}{s^{3}} \sum_{j}\left\{\frac{s^{2}}{4}\left(a_{j+1}^{\dagger}-a_{j}^{\dagger}\right)\left(a_{j+1}-a_{j}\right)\right. \\
-\frac{s}{16}\left[\left(a_{j}^{\dagger}\right)^{2}+a_{j+1}^{\dagger} a_{j}^{\dagger}+\left(a_{j+1}^{\dagger}\right)^{2}\right]\left[a_{j}^{2}+a_{j+1} a_{j}+a_{j+1}^{2}\right] \\
\left.+\frac{s}{16} a_{j+1}^{\dagger} a_{j}^{\dagger} a_{j+1} a_{j}-s^{2} a_{j}^{\dagger} a_{j}\right\}
\end{gathered}
$$

The model is solvable by algebraic Bethe Ansatz [7]. After simple manipulations Eq. (33) can be written as

$$
\begin{aligned}
& H_{F T T}=H_{B H} \\
& +V \sum_{j}\left[n_{j} n_{j+1}+\frac{1}{2} K_{j, j+1}^{2}+\left(n_{j}+n_{j+1}\right) K_{j, j+1}\right],
\end{aligned}
$$

where periodic boundary conditions have been considered (see Eqs.(31)); the parameters in $H_{B H}$ above are $\mu=\kappa(c \Delta)(c \Delta / 16+1) / 4, U=\kappa(c \Delta)^{2} / 64, t=\kappa c \Delta / 8$, and $V=\kappa(c \Delta)^{2} / 64$. Eq. (34) elucidates the relation between the Faddeev-Takhtadjan-Tarasov and BoseHubbard models. In the continuous limit, when lattice spacing $\Delta \rightarrow 0$ the Faddeev-Takhtadjan-Tarasov (and the Bose-Hubbard model as well) lattice model turns into the continuous Bose gas (11). 


\section{CONCLUSIONS.}

We considered several models of interacting bosons. Faddeev-Takhtadjan-Tarasov model and Izergin-Korepin model are different lattice models originally constructed from the Bose gas continuous field theory. For these models the lattice contributions do not destroy the integrability of the original field theory; instead the Bose Hubbard model contains lattice-effects leading to a non integrable dynamics. In the weak coupling limit these integrable Hamiltonians and the Bose-Hubbard Hamiltonian can be compared explicitly. In particular the model in Eq. (34) could serve exact studies of chains of Josephson junctions away from the degeneracy point [17]. The lattice effects vanish at low density: all these theories are integrable and described by the Bose gas with delta interaction. In this regime the known asymptotic of the Bose gas correlation functions can be used for physical systems captured by the Bose-Hubbard model (see 38] for a recent application). The Luttinger liquid field theory in Ref. [36] and the Bose gas are characterized by the same set of correlation functions where the spectral parameters $v_{J}, v_{F}, v_{N}$ are determined by [37] $\theta=2 \sqrt{v_{J} / v_{N}}, v_{F}=\sqrt{v_{N} v_{J}}$, and $v_{F}=\left[\epsilon_{0}^{\prime}(q)-\int_{-q}^{q} d u \epsilon_{0}^{\prime}(u) \tau(u)\right] /[\mathcal{Z}(q)]$ (see section II).

Discussions with R. Fazio, P. Kulish and A. Osterloh are acknowledged.

\section{APPENDIX}

The construction of integrable lattice models of interacting bosons is based on Quantum Inverse Scattering Method (QISM) [3]. Here we shall summarize some features of this method. The starting point of the QISM is a local quantum Lax operators $L_{i}(u)$ and a matrix $R(u)$, satisfying the Yang Baxter equation

$$
R(\phi) L_{i}(\zeta) \otimes L_{i}(\xi)=L_{i}(\xi) \otimes L_{i}(\zeta) R(\phi)
$$

The monodromy matrix

$$
T(\zeta)=L_{-N_{s}}(\zeta) L_{-N_{s}+1}(\zeta) \ldots L_{N_{s}}(\zeta)
$$

also fulfills the Yang-Baxter relation

$$
R(\phi) T(\zeta) \otimes T(\xi)=T(\xi) \otimes T(\zeta) R(\phi) .
$$

The transfer matrix is defined as $t(\zeta):=\operatorname{tr}_{(0)} T(\zeta)$ where $\operatorname{tr}_{(0)}$ means the trace in the auxiliary space. It is a generating functional of integrals of motion and of the Hamiltonian since it commutes with itself at different values of spectral parameters: $[t(\zeta), t(\xi)]=0(t(\zeta)$ is an invariant of the Yang-Baxter algebra). The quantum determinant 34

$$
\operatorname{det}_{q}\left(T(\zeta):=\operatorname{Tr} \mathcal{P} T(\zeta) \otimes T\left(e^{-\eta} \zeta\right),\right.
$$

is the analog of the Casimir operator of Lie algebras and it is another invariant of the Yang-Baxter algebra. In Eq. (37) $\mathcal{P}$ is the projector

$$
\mathcal{P}=R(\eta ; \eta):=\frac{1}{2 \cosh \eta}\left(\begin{array}{cccc}
0 & 0 & 0 & 0 \\
0 & e^{-\eta} & -1 & 0 \\
0 & -1 & e^{\eta} & 0 \\
0 & 0 & 0 & 0
\end{array}\right)
$$

To construct lattice integrable theories for the Bose gas two different procedures (somehow complementary) have been pursued. The first consists in keeping the $R$ matrix of the continuous theory unaltered and changing the Lax operators. Such procedure has been followed in the text above. One can also change both the $R$ and $L$ operators to simplify the final form of the Hamiltonian. This approach was initiated by P.P. Kulish et al. [8, 9] and developed in the papers [12, 13, 14], quantizing classical discretization on non-linear Schroedinger equation developed earlier by Ablowitz and Ladik [35]. For the Ablowitz-Ladik model the Lax operators and the Rmatrix [8, 9] are :

$$
L_{i}(\zeta):=\left(\begin{array}{cc}
\zeta & q_{i} \\
-q_{i}^{\dagger} & \zeta^{-1}
\end{array}\right), R(\phi ; \eta):=\left(\begin{array}{cccc}
1 & 0 & 0 & 0 \\
0 & b^{-} & c & 0 \\
0 & c & b^{+} & 0 \\
0 & 0 & 0 & 1
\end{array}\right)
$$

Here $\exp \phi=\zeta / \xi$ and $\zeta, \xi$ are spectral parameters,

$$
\begin{aligned}
& b^{ \pm}=\frac{e^{ \pm \eta} \sinh \phi}{\sinh (\phi-\eta)}, \\
& c=-\frac{\sinh \eta}{\sinh (\phi-\eta)},
\end{aligned}
$$

the quantity $\eta \in \mathbb{R}$ is a deformation parameter. The Hamiltonian of quantum Ablowitz-Ladik can be defined by

$$
H_{A L}=-\sum_{j=-N_{s}}^{N_{s}}\left[q_{j}^{\dagger} q_{j+1}+q_{j+1}^{\dagger} q_{j}-\alpha \log \left(1+q_{j}^{\dagger} q_{j}\right)\right]
$$

We shall see later that $\alpha$ doesn't play the role of coupling constant. In fact the potential and the kinetic energies commute (then the potential energy turns into a constant on the eigenstates of the kinetic term of (40)). The operators $q_{j}$ satisfy the following commutation relation:

$$
\left[q_{i}, q_{j}^{\dagger}\right]=\left(e^{2 \eta}-1\right)\left(1+q_{j}^{\dagger} q_{j}\right) \delta_{j}^{i}
$$

as follows the Yang-Baxter algebra. The Hamiltonian (40) can be rewritten using the trace identities. In particular the hopping term of the Ablowitz-Ladik model is

$$
-\sum_{j} q_{j} q_{j+1}^{\dagger}=\lim _{\zeta \rightarrow \infty} \frac{t(\zeta)-\zeta^{2 N_{s}+1}}{\zeta^{2 N_{s}-1}}
$$


and

$$
-\sum_{j} q_{j}^{\dagger} q_{j+1}=\lim _{\zeta \rightarrow 0} \frac{t(\zeta)-\zeta^{-2 N_{s}-1}}{\zeta^{1-2 N_{s}}},
$$

whereas the interaction is related to the quantum determinant

$$
\ln \operatorname{det}_{q}(T)=\sum_{i=-N_{s}}^{N_{s}} \log \left(1+q_{j}^{\dagger} q_{j}\right)+\left(2 N_{s}+1\right) \eta .
$$

In simple words: the hopping term (the kinetic energy) commutes with the interaction since the transfer matrix and the quantum determinant commute each other. Eigenvectors of the transfer matrix can be constructed by algebraic Bethe Ansatz (see Refs. [8, 12] or Chapter VII of the book [3]). Let us denote the off-diagonal element of monodromy matrix (35) by $C(\zeta)=T_{21}(\zeta)$. The eigenvectors of monodromy matrix and Hamiltonian can be written as

$$
|\chi\rangle=\prod_{k=1}^{n} C\left(\zeta_{k}\right)|0\rangle .
$$

Here $\mid 0>$ is the Fock vacuum $q_{j}|0\rangle=0$. The eigenvalue of monodromy matrix $\theta(\zeta)$ is

$$
\begin{aligned}
\theta(\zeta) & =\zeta^{2 N_{s}+1} e^{n \eta} \prod_{k=1}^{n} \frac{\sinh \left(\lambda-\lambda_{k}-\eta\right)}{\sinh \left(\lambda-\lambda_{k}\right)} \\
& +\zeta^{-2 N_{s}-1} e^{n \eta} \prod_{k=1}^{n} \frac{\sinh \left(\lambda-\lambda_{k}+\eta\right)}{\sinh \left(\lambda-\lambda_{k}\right)} .
\end{aligned}
$$

Here $\exp (\lambda)=\zeta$ (the same for $\exp \left(\lambda_{k}\right)=\zeta_{k}$ ). The variables $\lambda_{k}$ have to satisfy the Bethe equations

$$
e^{2\left(2 N_{s}+1\right) \lambda_{a}}=-\prod_{k=1}^{n} \frac{\sinh \left(\lambda_{a}-\lambda_{k}+\eta\right)}{\sinh \left(\lambda_{a}-\lambda_{k}-\eta\right)} .
$$

The vectors 45) are also eigenvectors of the logarithm of the quantum determinant (44) with eigenvalues equal to $\left(2 N_{S}+1\right) \eta$. The energy levels [ eigenvalues of the Hamiltonian] are

$$
2\left(1-e^{2 \eta}\right) \sum_{k=1}^{n} \cosh 2 \lambda_{k}
$$

The operators $q_{j}$ can be represented in terms of the standard Bose operators $\left[a_{n}, a_{m}^{\dagger}\right]=\delta_{m}^{n}$

$$
q=\sqrt{\frac{\exp 2 \eta\left(a^{\dagger} a+1\right)-1}{1+a^{\dagger} a}} a .
$$

The correlation functions of the model were evaluated in [13, 14].

[1] E.H.Lieb and W.Liniger Phys. Rev. 130, 1605 (1963).
[2] C.N.Yang, C.P. Yang. Journ. of Math. Phys. 10, 1115 (1969)

[3] V.E. Korepin, N.M. Bogoliubov, and A.G. Izergin, Quantum Inverse Scattering Method and Correlation Functions, (Cambridge Univ. Press, Cambridge 1993).

[4] V.E. Korepin and A.G. Izergin, Nucl. Phys. B 205, 401 (1982).

[5] V.E. Korepin and A.G. Izergin, Doklady Akad. Nauk 259, 76, (1981).

[6] A. Kundu and O. Ragnisco, J. Phys. A 27, 6335 (1994).

[7] V.O.Tarasov, L.A. Takhadzhyan and L.D. Faddeev, Theoretical and Mathematical Physics 57, 163 (1983).

[8] P. Kulish, Lett. Math. Phys. 5, 191 (1981).

[9] V.S. Gerdzikov, M.I. Ivanov, and P.P.Kulish, J. Math. Phys. 25, 25 (1984).

[10] N.M. Bogoliubov, R.K.Bullough, Phys. Lett. A 168, 264 (1992)

[11] N.M. Bogoliubov, R.K.Bullough, Journ. Phys. A: Math. Gen. 25 , 4057, (1992)

[12] N.M. Bogoliubov, R.K.Bullough and G.D.Pang. Phys. Rev B 47, 11495 (1993)

[13] N.M. Bogoliubov, R.K.Bullough and Timonen, Phys. Rev. Lett. 72，3933 (1994)

[14] N.M. Bogoliubov, A.G.Izergin and N.A. Kitanine, Nucl. Phys. B 516 , 501, 1998

[15] F.D.M. Haldane, Phys. Lett. A 80, 281 (1980)

[16] M.P.A. Fisher, P.B. Weichman, G. Grinstein, and D.S. Fisher, Phys. Rev. B 40, 546 (1989).

[17] R. Fazio and H. van der Zant, Phys. Rep. 355, 235 (2001).

[18] D. Jaksch, C. Bruder, J.I. Cirac, C.W. Gardiner, and P. Zoller, Phys. Rev. Lett. 81, 3108 (1998).

[19] J. J. Garcia-Ripoll, M. A. Martin-Delgado, and J. I. Cirac, cond-mat/0404566

[20] M. Greiner et al., Nature 415, 39 (2002).

[21] G.G. Batrouni, et al., Phys. Rev. Lett. 89, 117203 (2002).

[22] M. Girardeau, J. Math. Phys. 1, 516 (1960); Phys. Rev. 139, B500 (1965).

[23] E.H. Lieb, R. Seiringer, and J. Yngvason, Phys. Rev. Lett. 91, 150401 (2003).

[24] F.D.M. Haldane, Phys. Lett. A 81, 545 (1981); T.C. Choy, Phys. Lett. A 80, 49 (1980); T.C. Choy and F.D.M. Haldane Phys. Lett. A 90, 83 (1990).

[25] J.-B. Bru and T. Dorlas, J. Stat. Phys. 113, 177 (2003).

[26] B. Sutherland, Exactly solvable problems in condensed matter and relativistic field theory, Lecture notes in physics 242, eds S. Shastry, S.S. Jha, and V. Singh (Springer Berlin, 1985).

[27] A.R. Kolovsky and A. Buchleitner, cond-mat/0403213

[28] G. Montambaux et al., Phys. Rev. Lett. 70, 497 (1993).

[29] N.M. Bogoliubov, A.G. Izergin, and N.Yu. Reshetikhin, J. Phys. A 20, 5361 (1987).

[30] A.P. Polychronakos, in Topological aspects of low dimensional systems, Les Houches lectures notes (EDP, Springer, 1998).

[31] R.W. Richardson and N. Sherman, Nucl. Phys. 52, 221 (1964); 52, 253 (1964).

[32] L. Amico, A. Di Lorenzo, and A. Osterloh, Phys. Rev. Lett 86, 5759 (2001); Nucl. Phys. B 614, 449 (2001).

[33] J. Dukelsky, S. Pittel, and G. Sierra, nucl-th/0405011

[34] A.G. Izergin and V.E. Korepin, Sov. Phys. Dokl. 26 (7), 653 (1981).

[35] Ablowitz and Ladik, J. Math. Phys. 16, 598 (1975); ibid. 17, 1011 (1976). 
[36] F.D.M. Haldane, Phys. Rev. Lett. 47, 1840 (1981).

[37] F.D. Haldane, Phys. Lett. 81, 153 (1981).

[38] C. Kollath, U. Schollwöck, J. von Delft, and W. Zwerger, 